\title{
A DOMAIN SPLITTING METHOD FOR HEAT CONDUCTION PROBLEMS IN COMPOSITE MATERIALS
}

\author{
FRIEDRICH KARL HEBEKER ${ }^{1}$
}

\begin{abstract}
We consider a domain decomposition method for some unsteady heat conduction problem in composite structures. This linear model problem is obtained by homogenization of thin layers of fibres embedded into some standard material. For ease of presentation we consider the case of two space dimensions only. The set of finite element equations obtained by the backward Euler scheme is parallelized in a problem-oriented fashion by some noniterative overlapping domain splitting method, eventually enhanced by inexpensive local iterations to reduce the overlap. We present a detailed convergence analysis of this algorithm which is particularly well appropriate to handle fibre layers of nonlinear material. Special emphasis is to take into account the specific regularity properties of the present mathematical model. Numerical experiments show the reliability of the theoretical predictions.
\end{abstract}

Mathematics Subject Classification. 80A22, 65M.

Received: April 15, 1999.

\section{INTRODUCTION}

Composite materials offer a wide range of practical applications. Of particular interest are structures where some 'adaptive substance' (shape-memory alloy, etc.) is embedded as fibres into some 'standard' material. Such structures lead to new and challenging problems of mathematical and numerical analysis. In the present note we dwell on the specific task of large demand of computing power required to solve even moderately complex problems of this field. We introduce a (linear) model problem for analyzing unsteady effects in composite materials and investigate problem-adapted parallel algorithms to solve this. These algorithms are based on the noniterative overlapping domain splitting method, a universal approach designed to handle mathematical models of realistic complexity in a problem-oriented manner on parallel computers.

In the present analysis we restrict ourselves to a relatively simple but nevertheless typical model problem, which is obtained by homogenization of thin layers of fibres and dimension reduction (cf. [7,13]). Our model and its numerical analysis is two-dimensional and linear (for ease of presentation), but the methods are mostly aimed for realistic nonlinear problems in three space dimensions. We point out that similar methods are applicable to problems of layered semiconductors, $c f$. [11].

We briefly motivate the subsequent mathematical model, see [13] for details. The thin fibres (diameter $0<\varepsilon \ll 1$ of the cross section) are packed along planes $y=c$ in the $\mathbb{R}^{3}$ (see Fig. 1). We consider mean values of

\footnotetext{
Keywords and phrases. Fibre layers of adaptive material, homogenization, heat conduction, finite element method, noniterative overlapping domain decomposition.

1 Fachbereich Mathematik, Justus-Liebig-Universität Gießen, Arndtstr. 2, 35392 Gießen, Germany.

e-mail: friedrich.k.hebeker@math.uni-giessen.de
} 


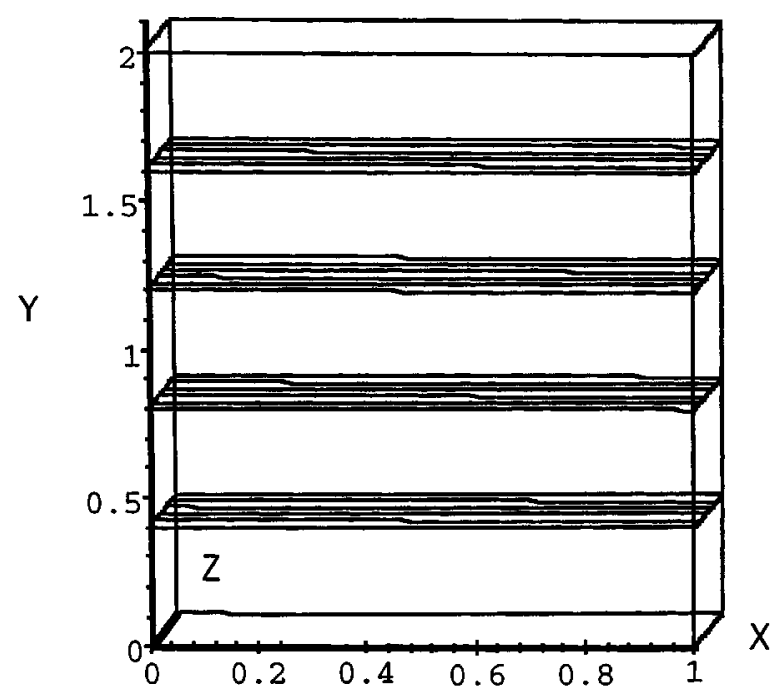

FiguRE 1. Layers of fibres, homogenized to planes.

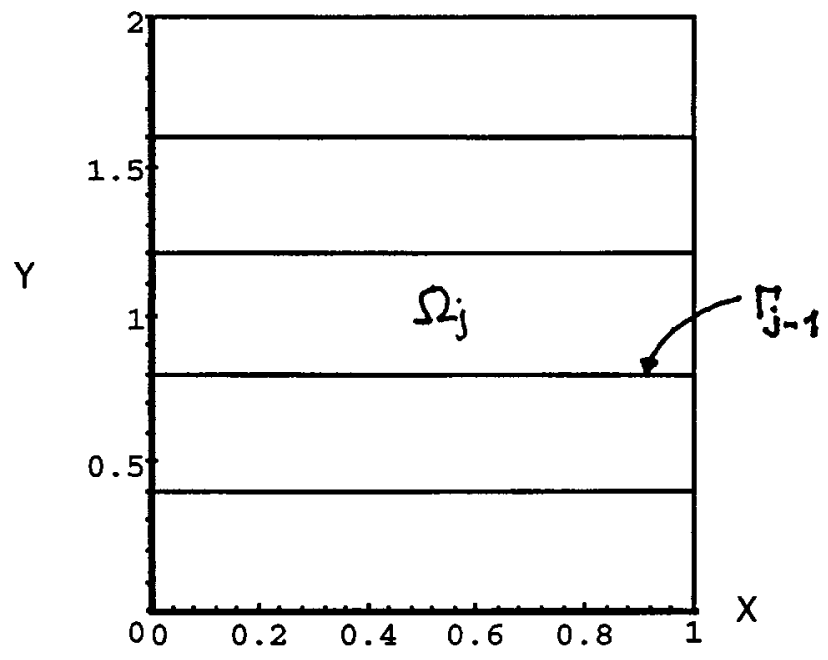

Figure 2. Two-dimensional domain, subdomains $\Omega_{j}$ and interfaces $\Gamma_{j}$.

the heat equation in control volumes $V$ of side length $\varepsilon$ (divided into two equally sized parallelepipeds by such planes). Assuming continuity of all physical quantities but the normal derivative of the temperature $u$ we get

$$
\int_{V}\left(u_{t}+\operatorname{div} q-f\right) \mathrm{d} V \approx \varepsilon^{3}\left(u_{t}+q_{x}^{(1)}+q_{z}^{(3)}-f\right)+\varepsilon^{2}\left[q^{(2)}\right]=0
$$

where $q=\left(q^{(1)}, q^{(2)}, q^{(3)}\right)^{T}=-\kappa \nabla u$ denotes the heat flux and $[q]:=q_{\text {upper }}-q_{\text {lower }}$ the jump when passing through the interface. We finally assume that none of all quantities depends on the $z$-variable, thus resulting in a two-dimensional model (Fig. 2), particularly in the equation (3) on the surface.

We thus consider a rectangular broken domain $\Omega_{*}:=\cup_{j=0}^{J} \Omega_{j}$ in the $(x, y)$-plane, consisting of $J+1$ open stripes $\Omega_{j}:=(0,1) \times\left(y_{j-1}, y_{j}\right)$. The stripes $\Omega_{j}$ are separated by $J$ interfaces $\Gamma_{j}:=(0,1) \times\left\{y_{j}\right\}$, we further 
abbreviate $\Gamma:=\cup_{j=1}^{J} \Gamma_{j}$. The boundary $\partial \Omega_{*}$ of the domain decomposes into a closed manifold $\Sigma$ of edges and the set $\Gamma$ of interfaces:

$$
\partial \Omega_{*}=\Sigma+\Gamma .
$$

Let further $\Omega$ denote the total domain (with boundary $\Sigma$ ) defined as

$$
\Omega:=\Omega_{*}+\Gamma .
$$

Let $n=\left(n_{x}, n_{y}\right)$ denote the outer normal on $\Sigma$ and also the upper normal on the interfaces. Occasionally, the trace on the interface of some (continuous) function $v$ on $\Omega$ is denoted by $\bar{v}$. - We are looking for a scalar function $u$ solving the heat equation

$$
u_{t}-\kappa \Delta u=f \text { in } \Omega_{*}
$$

in a time interval $(0, T]$, with a given constant heat conductivity $\kappa>0$ and a given function $f$. Due to continuity of $u$ (and of its tangential derivative)

$$
[u]=\left[u_{x}\right]=0 \quad \text { on } \Gamma .
$$

However, the normal derivative may jump, thus evoking a dynamical process on the interfaces modelled by a differential equation

$$
\bar{\rho} u_{t}-\bar{\kappa} u_{x x}=\bar{f}+\left[\kappa u_{y}\right] \text { on } \Gamma
$$

with given physical quantities $\bar{\rho}, \bar{\kappa}$, and $\bar{f}$ (depending on the geometry of the fibres and their environment and obtained by homogenization as indicated above). In the present analysis we restrict ourselves to normalized $\bar{\rho} \equiv 1$ and also $\bar{\kappa} \equiv$ const. $>0$. This evolution problem is supplemented by boundary and initial conditions

$$
\begin{array}{ll}
u=0 & \text { on } \Sigma \\
u=u_{0} & \text { at } t=0 .
\end{array}
$$

This problem serves as a linear model for heat conduction in composite materials. It should be pointed out that real-life applications are generally modelled by nonlinear physical quantities, particularly $\bar{f}, \bar{\kappa}$. But all present methods are actually designed to handle nonlinear problems. Several remarks concerning this are included into the present paper. - For a different numerical approach to composite materials, by using finite elements specially adapted to microstructures, see [6].

We introduce 'broken' spaces and norms (subindexed by a ' $*$ '). Let

$$
H_{*}^{m}(\Omega):=\prod_{j} H^{m}\left(\Omega_{j}\right)
$$

denote the Sobolev space of all functions that are square integrable over all $\Omega_{j}$ along with all their weak partial derivatives up to order $m . H_{*, 0}^{m}(\Omega)$ is the subspace of functions with zero trace on $\Sigma$. We use the 'broken' Sobolev norm

$$
\|v\|_{*, m}:=\left(\sum_{j}\|v\|_{m, \Omega_{j}}^{2}\right)^{1 / 2}
$$

on $H_{*}^{m}(\Omega)$ and $H_{*, 0}^{m}(\Omega)$, and also $|v|_{*, m}:=\left(\sum_{j}|v|_{m, \Omega_{j}}^{2}\right)^{1 / 2}$ the seminorm (or norm) of the highest derivatives on $H_{*}^{m}(\Omega)$ (or $H_{*, 0}^{m}(\Omega)$, resp). The $L^{2}(\Omega)$-norm is shorthand $\|$.$\| , the L^{2}(\Omega)$-scalar product (.,.). We define 
these norms and scalar products on the interface $\Gamma$ analogously. All these quantities are often indexed to indicate the domain of integration: for instance, $(u, v)_{G}:=\int_{G} u v \mathrm{~d} G$.

Following [13], we formulate this evolution problem as an evolution system of variational equations. We introduce the space

$$
X:=\left\{u \in H_{*, 0}^{1}(\Omega) \cap H_{0}^{1}(\Gamma):[u]=0 \text { on } \Gamma\right\}
$$

which coincides with $H_{0}^{1}(\Omega) \cap H_{0}^{1}(\Gamma)$. Endowed with the norm

$$
\|u\|_{X}:=\left(\|\nabla u\|^{2}+\left\|u_{x}\right\|_{\Gamma}^{2}\right)^{1 / 2}
$$

it serves as the energy space of our problem. The evolution problem is formulated in weak form as follows: we are looking for $u(t) \in X$ satisfying

$$
\left(u_{t}, \varphi\right)+\left(u_{t}, \varphi\right)_{\Gamma}+(\kappa \nabla u, \nabla \varphi)+\left(\bar{\kappa} u_{x}, \varphi_{x}\right)_{\Gamma}=(f, \varphi)+(\bar{f}, \varphi)_{\Gamma}
$$

in the time interval $(0, \mathrm{~T})$ for all $\varphi \in X$, and the weak initial condition

$$
(u(0), \varphi)+(u(0), \varphi)_{\Gamma}=\left(u_{0}, \varphi\right)+\left(u_{0}, \varphi\right)_{\Gamma}
$$

for all $\varphi \in L^{2}(\Omega) \cap L^{2}(\Gamma)$. Existence and uniqueness of a global weak solution of this heat conduction problem is proved by standard arguments ( $c f$. [19], also [13]) based upon the basic properties of the corresponding stationary operator, see [9].

Since the nonlinearities often enforce very small time steps, the variational form is discretized by a backward Euler scheme, using linear finite elements in space. For typical nonlinear problems, iteration on the resulting system of equations is quite costly, as has been shown by [13] in case of the Landau-Ginzburg nonlinearity, where the algorithm has been parallelized (in a data-oriented manner) in order to handle the large data set. In the present paper we introduce a problem-oriented parallel algorithm. The resulting noniterative overlapping domain splitting scheme extends that introduced and first investigated by Kuznetsov [14,16] in case of standard parabolic equations. It is based on the exponential decay of the discrete Green's function. In contrast to the algebraic proof by Kuznetsov, a different one, solely based on variational arguments, has been presented by Blum, Lisky, and Rannacher [1]. Moreover, in the latter paper the actual parallel efficiency of this algorithm has been substantiated by numerical tests on a parallel computer (see also [12] for a detailed parallel analysis). Later, this method has been extended to convection-diffusion problems by $[10,15,18]$ as well as to mixed finite element formulations by [3].

The present paper is organized as follows. The methods of discretization are carried out and analyzed in Section 2. For solving in parallel the resulting problem arising in each time step we investigate a noniterative overlapping domain splitting method. The resulting algorithm and the corresponding convergence analysis is topic of Section 3. In Section 4 we present the proof of the convergence theorem and in Section 5 that of the basic exponential decay lemma. In the concluding Section 6 we present several numerical results of a test example showing the reliability of our theoretical predictions.

As a peculiar point we show that, in some cases where the overlap would be inconvenient or unacceptably large, very few local iterations of the present algorithm to improve on the boundary data of the artificial inner boundaries would help to reduce the overlap drastically. Note that, to this end, local problems are to be solved only, consequently the additional expenses are low. - Moreover, in case of a test example, it turns out that the domain splitting algorithm nearly 'scales' with the number of substructures. This favorable effect has also been observed for standard heat problems, [1]. Nevertheless, we are unable to decide here whether this effect is an artifact of our test example or is inherent in the algorithm. This important point is left to further investigations, ideally by treating some physical example (e.g. [13]) and employing a parallel computer. 


\section{Discretization}

Here we introduce an implicit scheme with linear finite elements to discretize the model problem. The elements are either triangles or quadrilaterals. For the domain splitting scheme we require a quasi-uniform family of admissible triangulations $\mathcal{T}_{h}$ of the total domain $\Omega$. Let $X_{h} \subset X$ denote the space of discrete functions $U$ which are continuous in $\bar{\Omega}$ and (with respect to $\mathcal{T}_{h}$ ) piecewise (bi-)linear ansatz functions, vanishing on $\Sigma$. Let $\left\{t_{n}\right\}$ (with $t_{n}:=n \cdot k$ ) denote an equidistant time mesh, with $n \leq N\left(N\right.$ the largest integer not greater $\left.\frac{T}{k}\right)$ and mesh size $k$, and $v^{n}:=v\left(t_{n},.\right)$.

We are looking for a set $\left\{U^{n}\right\}$ of discrete functions $U^{n} \in X_{h}$ as approximation to $u\left(t_{n},.\right)$, obtained by replacing the time derivatives in the variational form by $u_{t} \approx \frac{1}{k}\left(U^{n+1}-U^{n}\right)$. Moreover, all diffusive terms and given functions are discretized implicitly according to the backward Euler scheme.

The algorithm starts with the projections as initial data

$$
\left(U^{0}, \Phi\right)+\left(U^{0}, \Phi\right)_{\Gamma}=\left(u_{0}, \Phi\right)+\left(u_{0}, \Phi\right)_{\Gamma}
$$

for all $\Phi \in X_{h}$. It proceeds iteratively as

Algorithm 1 (Discrete algorithm). Given $U^{n} \in X_{h}$. Solve the discrete evolution equations

$$
\left(U^{n+1}, \Phi\right)+\left(U^{n+1}, \Phi\right)_{\Gamma}+k\left(\kappa \nabla U^{n+1}, \nabla \Phi\right)+k\left(\bar{\kappa} U_{x}^{n+1}, \Phi_{x}\right)_{\Gamma}=L^{n}(\Phi)
$$

for all $\Phi \in X_{h}$, inductively on all time levels $n+1$. Here the functionals are

$$
L^{n}(\Phi):=\left(U^{n}, \Phi\right)+\left(U^{n}, \Phi\right)_{\Gamma}+k\left(f^{n+1}, \Phi\right)+k\left(\bar{f}^{n+1}, \Phi\right)_{\Gamma}
$$

where $f^{n+1}:=f\left(t_{n+1},.\right)$, etc.

Some remarks concerning nonlinear terms are in order.

Remark 1. Nonlinear terms, for instance with $\bar{f}:=\bar{f}(u)$, should be discretized explicitly. Eventually, this approximation should be corrected by a certain number of inner iterations for each time step. This is generally an expensive procedure, but it should be noted that, with the domain splitting method below, these inner iterations have actually to be carried out on small subdomains (which include the interfaces) only. This seems to be a peculiar advantage of our domain decomposition method.

Concerning this discretization scheme we have an optimal convergence result. It is based on the following elliptic regularity property of the stationary operator. (For a more detailed analysis of this operator see [9].)

Lemma 1. Assume $f \in L^{2}(\Omega)$ and $\bar{f} \in L^{2}(\Gamma)$. Then the solution $u \in X$ of the variational equation

$$
(\kappa \nabla u, \nabla \varphi)+\left(\bar{\kappa} u_{x}, \varphi_{x}\right)_{\Gamma}=(f, \varphi)+(\bar{f}, \varphi)_{\Gamma}
$$

for all $\varphi \in X$, is in fact in $H_{*}^{2}(\Omega)$ and in $H^{2}(\Gamma)$. Moreover

$$
\|u\|_{*, 2}+\|u\|_{2, \Gamma} \leq \operatorname{const.}\left(\|f\|+\|\bar{f}\|_{\Gamma}\right)
$$

holds.

Proof. It suffices to show the assertion for the highest derivatives. We start from the representation formula (e.g., [5], p. 201)

$$
|u|_{2, \Omega_{j}}^{2}=\|\Delta u\|_{\Omega_{j}}^{2}-2 \int_{\partial \Omega_{j}}\left(u_{x x} \tilde{n}_{y}-u_{x y} \tilde{n}_{x}\right) u_{y} \mathrm{~d} s
$$


where $\tilde{n}=\left(\tilde{n}_{x}, \tilde{n}_{y}\right)$ is the outer normal on $\partial \Omega_{j}$. On $\partial \Omega_{j} \cap \Sigma$ we have $u_{y}=0$ so that the boundary integral vanishes on this part of the boundary. On the other hand, the second term of the boundary integral disappears on the interface. Sum then over all subdomains to yield

$$
|u|_{*, 2}^{2}=\|\Delta u\|^{2}+2 \int_{\Gamma} u_{x x}\left[u_{y}\right] \mathrm{d} s .
$$

Inserting now the steady differential equations we get

$$
\begin{aligned}
|u|_{*, 2}^{2}+\frac{2 \bar{\kappa}}{\kappa}|u|_{2, \Gamma}^{2} & =\left\|\frac{1}{\kappa} f\right\|^{2}-\frac{2}{\kappa} \int_{\Gamma} u_{x x} \bar{f} \mathrm{~d} s \\
& \leq c\|f\|^{2}+\varepsilon|u|_{2, \Gamma}^{2}+c_{\varepsilon}\|\bar{f}\|_{\Gamma}^{2} .
\end{aligned}
$$

Choosing $\varepsilon$ sufficiently small the assertion follows.

This implies a convergence theorem, under realistic assumptions on the data.

Theorem 1 (Convergence of the discrete solutions). Assume that

$$
\begin{aligned}
& u \in L^{\infty}\left(0, T ; H_{*}^{2}(\Omega) \cap H^{2}(\Gamma)\right) \\
& u_{t} \in L^{2}\left(0, T ; H_{*}^{2}(\Omega) \cap H^{2}(\Gamma)\right) \\
& u_{t t} \in L^{2}\left(0, T ; L^{2}(\Omega) \cap L^{2}(\Gamma)\right) .
\end{aligned}
$$

Then the sequence of discrete solutions converges as $h, k \rightarrow 0$ :

$$
\left\|u\left(t_{n}, .\right)-U^{n}\right\|+\left\|u\left(t_{n}, .\right)-U^{n}\right\|_{\Gamma} \leq \text { const. }\left(h^{2}+k\right)
$$

for all $n=0, \ldots, N$.

Proof. Let $W(t) \in X_{h}$ denote the pointwise Galerkin projection of $u(t)$, then

$$
(\kappa \nabla(u(t)-W(t)), \nabla V)+\left(\bar{\kappa}\left(u_{x}(t)-W_{x}(t)\right), V_{x}\right)_{\Gamma}=0
$$

holds for all $V \in X_{h}$. Lemma 1 implies an approximation result in the sense of Aubin and Nitsche

$$
\left\|\frac{\partial^{k}}{\partial t^{k}}(u(t)-W(t))\right\|+\left\|\frac{\partial^{k}}{\partial t^{k}}(u(t)-W(t))\right\|_{\Gamma} \leq c h^{2}\left(\left\|\frac{\partial^{k} u(t)}{\partial t^{k}}\right\|_{*, 2}+\left\|\frac{\partial^{k} u(t)}{\partial t^{k}}\right\|_{2, \Gamma}\right)
$$

for all $k$. It should be noticed that the broken $H^{2}$-estimate of $\frac{\partial^{k} u(t)}{\partial t^{k}}$ just suffices. The difference $W-U$ is then bounded via a differential inequality. The remainder of the proof is quite standard and omitted here (see [17], e.g.).

Note that nearly such convergence order (up to a logarithmic factor) can be established even for general smooth interfaces nonmatching with the elements, using the recent analysis by [4] (cf. [8] for an a posteriori analysis).

\section{The DOMAIN SPLitTing ALGORITHM}

We observe that in composite materials the differential operators are often simple in the (large) stripes, but intricate (generally nonlinear) on the interfaces only. Hence some nonlinearity were, in a sense, concentrated on small regions in the domain. This is the leading motivation for our concrete domain splitting scheme. 


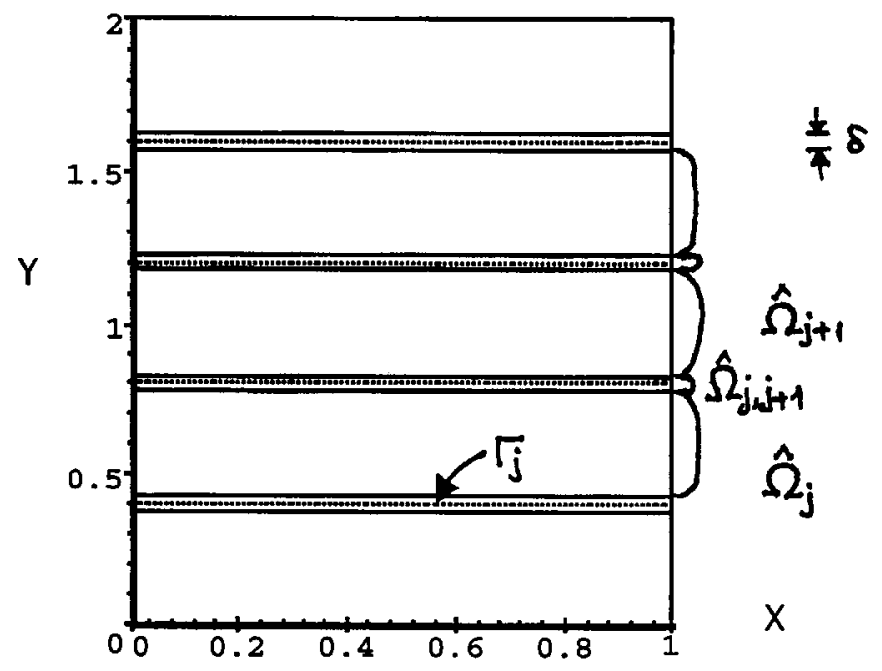

FiguRE 3. Nonoverlapping domain decomposition (interfaces $\Gamma_{j}$ dotted).

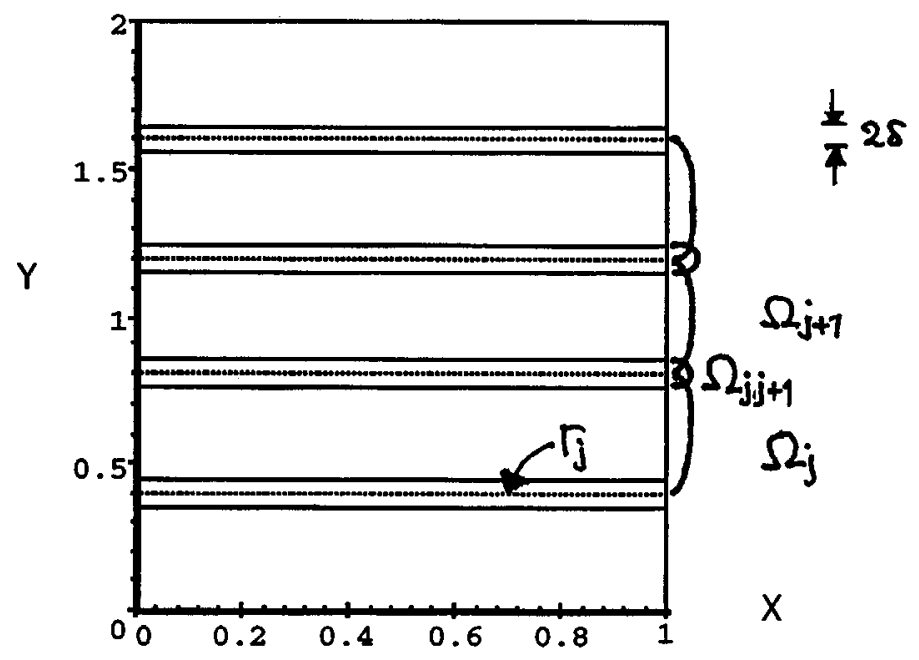

FiguRE 4. Overlapping domain decomposition (interfaces $\Gamma_{j}$ dotted).

We start with an auxiliary nonoverlapping decomposition of the domain. See Figure 3. Let the parameter $0<\delta \ll 1$ be given. We introduce the nonoverlapping domain splitting

$$
\Omega^{*}=\bigcup_{j=0}^{J} \hat{\Omega}_{j} \cup \bigcup_{j=0}^{J-1} \hat{\Omega}_{j, j+1}
$$

into large subdomains, defined as $\hat{\Omega}_{0}:=(0,1) \times\left(0, y_{1}-\frac{\delta}{2}\right)$ and for $j=1 \ldots J-1 \hat{\Omega}_{j}:=(0,1) \times\left(y_{j}+\frac{\delta}{2}, y_{j+1}-\frac{\delta}{2}\right)$ and finally $\hat{\Omega}_{J}:=(0,1) \times\left(y_{J}+\frac{\delta}{2}, y_{J+1}\right)$, and into small subdomains $\hat{\Omega}_{j, j+1}:=(0,1) \times\left(y_{j+1}-\frac{\delta}{2}, y_{j+1}+\frac{\delta}{2}\right)$. Now 
by enlarging all subdomains we get the overlapping domain splitting, see Figure 4,

$$
\Omega^{*}=\bigcup_{j=0}^{J} \Omega_{j} \cup \bigcup_{j=0}^{J-1} \Omega_{j, j+1}
$$

into the original large subdomains $\Omega_{j}$ and some small subdomains $\Omega_{j, j+1}:=(0,1) \times\left(y_{j+1}-\delta, y_{j+1}+\delta\right)$. The width of the overlapping stripes is always $\delta$. For simplicity, we assume that all the lines $(0,1) \times\left\{y_{j} \pm \delta\right\}$ consist of edges of the elements. If not, the sets $\Omega_{j, j+1}$ had to be modified appropriately. Let $X_{h}\left(\Omega_{j}\right)$ or $X_{h}\left(\Omega_{j, j+1}\right)$ denote those subsets of $X_{h}$ consisting of functions with support contained in the indicated subdomains.

The noniterative overlapping domain splitting scheme to treat the linear system (10) in parallel is now to solve the differential equation on all overlapping subdomains, where the boundary data are obtained by extrapolation from the previous time step.

Algorithm 2 (Domain splitting algorithm). Assume that the data $V^{n}$ and the corresponding functional $L^{n}$ are already computed. Then solve the following set of problems on the subdomains:

1. On all $\Omega_{j}$, solve for $V \equiv V_{j}^{n+1}$ piecewise linear:

$$
(V, \Phi)+k(\kappa \nabla V, \nabla \Phi)=L^{n}(\Phi)
$$

for all $\Phi \in X_{h}\left(\Omega_{j}\right)$, according to the initial data $V=V^{n}$ at $t=t_{n}$ and the boundary data $V=V^{n}$ on $\partial \Omega_{j}$.

2. On all $\Omega_{j, j+1}$, solve for $V \equiv V_{j, j+1}^{n+1}$ piecewise linear:

$$
(V, \Phi)+(V, \Phi)_{\Gamma_{j}}+k(\kappa \nabla V, \nabla \Phi)+k\left(\bar{\kappa} V_{x}, \Phi_{x}\right)_{\Gamma_{j}}=L^{n}(\Phi)
$$

for all $\Phi \in X_{h}\left(\Omega_{j, j+1}\right)$, according to the initial data $V=V^{n}$ at $t=t_{n}$ and the boundary data $V=V^{n}$ on $\partial \Omega_{j, j+1}$.

3. Finally, from the patchwise solutions $\{V\}$, a global single-valued vector function $V^{n+1}:=\mathcal{C}\{V\} \in X_{h}$ is constructed through a suitable averaging process.

Concerning this algorithm several remarks are in order.

\section{Remark 2.}

(a) Concerning the averaging process, we fix the operator $\mathcal{C}$ by setting $\mathcal{C}\{V\}=V_{j}^{n+1}$ in knots of $\overline{\hat{\Omega}_{j}}$ and $\mathcal{C}\{V\}=V_{j, j+1}^{n+1}$ in those of $\hat{\Omega}_{j, j+1}$, for instance. This guarantees $L^{2}$-stability of the operator $\mathcal{C}$ with constant 1 ,

$$
\|\mathcal{C}\{V\}\|^{2} \leq \sum_{j}\left(\|V\|_{\hat{\Omega}_{j}}^{2}+\|V\|_{\hat{\Omega}_{j, j+1}}^{2}\right) .
$$

Evidently, $\mathcal{C}\{V\}=V$ for a single-valued function $V$.

(b) It is a typical advantage of this problem-oriented parallel algorithm that, depending on the physical and geometrical problem and on the computer architecture, there are many ways to realize this algorithm practically on a parallel computer. For instance, all subproblems under 1. are independent and essentially of the same complexity. Thus, in a first step all these subproblems may be solved in parallel. In the second step then all subproblems under 2. may be solved in parallel. In this approach the boundary data of 2. might be replaced by those approximations obtained from the first step. Moreover, if these local problems were nonlinear, it makes sense to carry out nonlinear iterations on just these (small) subdomains which again are done in parallel.

(c) In case the number of processors exceeds that of the subdomains there are several ways to improve on the load balancing. For instance, all subdomains may be further subdivided into smaller pieces. Alternatively, as a unified step, all subdomains may be treated in parallel if appropriate, depending on the geometry and the nonlinearities. 
The main question is how to choose the parameter $\delta$. For reasons of economy it should be not too large. It has been shown by Kuznetsov [14] in case of parabolic equations that the number $L$ of overlapping elements (where $\delta=L h$ ) increases logarithmically only with the parameters of discretization. This fact is based on the exponential decay of the discrete Green's function. Blum, Lisky, and Rannacher [1] refer to an amount of $L=3$ to 4 as sufficient in case of standard parabolic equations. In case $\delta$ appears too large, Algorithm 2 should be pre-iterated to decrease $\delta$ drastically (see Sect. 6 below).

Thus we will prove as the main result of the present analysis

Theorem 2. Assume that the overlap $\delta$ satisfies

$$
\delta \geq \delta_{0} h \log \frac{1}{k}
$$

with a certain constant $\delta_{0}$. Then the domain decomposition algorithm is numerically stable, and for the difference between the domain splitting approximation $V^{n}$ and the discrete solution $U^{n}$ there holds the estimate

$$
\left\|U^{n}-V^{n}\right\|+\left\|U^{n}-V^{n}\right\|_{\Gamma} \leq \text { const. } k^{3 / 2} .
$$

This shows that the additional error due to domain splitting is small ( $c f$. Theorem 1) provided a sufficient (moderately large) amount of overlap is used. The number of overlapping elements increases like log $\frac{1}{k}$ at most. The proof uses the fact that pollution on the artificial boundaries decreases exponentially in space. The latter effect is stated as

Lemma 2 (Exponential decay lemma). Let $U$ piecewise linear in $\Omega_{j}$ (or in $\Omega_{j, j+1}$, resp.), solving either

$$
(U, \Phi)+k(\kappa \nabla U, \nabla \Phi)=0
$$

for all $\Phi \in X_{h}\left(\Omega_{j}\right)$, or

$$
(U, \Phi)+(U, \Phi)_{\Gamma_{j}}+k(\kappa \nabla U, \nabla \Phi)+k\left(\bar{\kappa} U_{x}, \Phi_{x}\right)_{\Gamma_{j}}=0
$$

for all $\Phi \in X_{h}\left(\Omega_{j, j+1}\right)$, resp., according to the boundary conditions

$$
U=F \quad \text { on } \partial \Omega_{j} \quad \text { (or on } \partial \Omega_{j, j+1} \text {, resp.). }
$$

Here $F$ are given piecewise linear functions on $\Omega_{j}$ or $\Omega_{j, j+1}$, resp. Then the following estimates hold:

$$
\begin{aligned}
\|U\|_{\hat{\Omega}_{j}}^{2} & \leq c e^{-\gamma \delta / \max \{\sqrt{k \kappa}, h\}}\left(\|F\|_{\Omega_{j}}^{2}+k \kappa\|\nabla F\|_{\Omega_{j}}^{2}\right) \\
\|U\|_{\hat{\Omega}_{j, j+1}}^{2}+\|U\|_{\Gamma_{j}}^{2} & \leq c e^{-\gamma \delta / \max \{\sqrt{k \kappa}, h\}}\left(\|F\|_{\Omega_{j, j+1}}^{2}+\|F\|_{\Gamma_{j}}^{2}+k \kappa\|\nabla F\|_{\Omega_{j, j+1}}^{2}+k \bar{\kappa}\left\|F_{x}\right\|_{\Gamma_{j}}^{2}\right)
\end{aligned}
$$

where $c, \gamma$ are positive constants, not depending on $\delta, h, k, j$ (but depend on $\kappa, \bar{\kappa}$ ).

We will prove the theorem in the following section and after that the exponential decay lemma.

\section{Proof of the Theorem 2}

The proof of estimate (20) extends the approach by [1]. We bound

$$
E^{n}:=\left\|U^{n}-V^{n}\right\|^{2}+\left\|U^{n}-V^{n}\right\|_{\Gamma}^{2}
$$


by induction over the time levels $t_{n}$. We assume without loss of generality

$$
1 \leq \sigma:=\frac{k \kappa}{h^{2}} \leq c
$$

To keep clear, we write $V_{j}^{n+1}, V_{j, j+1}^{n+1}$ for the patchwise solutions obtained with Algorithm 2.

According to the properties of the averaging operator $\mathcal{C}$ (see Rem. 2) we have that

$$
\begin{aligned}
E^{n+1} & =\left\|\mathcal{C}\left\{U^{n+1}-V\right\}\right\|^{2}+\left\|U^{n+1}-V^{n+1}\right\|_{\Gamma}^{2} \\
& \leq \sum_{j}\left(\left\|U^{n+1}-V_{j}^{n+1}\right\|_{\hat{\Omega}_{j}}^{2}+\left\|U^{n+1}-V_{j, j+1}^{n+1}\right\|_{\hat{\Omega}_{j, j+1}}^{2}+\left\|U^{n+1}-V^{n+1}\right\|_{\Gamma_{j}}^{2}\right) .
\end{aligned}
$$

We now introduce a field $\tilde{V}^{n+1}$ obtained by one step of the (global) Algorithm 1 (10), but with initial data $V^{n}$ instead of $U^{n}$. Then $\tilde{V}^{n+1}-V_{j}^{n+1}$ solves (21) in $\Omega_{j}$ and $\tilde{V}^{n+1}-V_{j, j+1}^{n+1}$ solves (22) in $\Omega_{j, j+1}$, according to the boundary data $\tilde{V}^{n+1}-V^{n}$. Consequently, by Young's inequality (with a positive $\varepsilon$ to be specified later) and Lemma 2, combined with the inverse inequality of quasi-uniform partitions and (25), we obtain for the large subdomains by setting $\delta=L h$ ( $L$ the number of rows of overlapping elements)

$$
\begin{aligned}
\left\|U^{n+1}-V_{j}^{n+1}\right\|_{\hat{\Omega}_{j}}^{2} & \leq(1+\varepsilon)\left\|U^{n+1}-\tilde{V}^{n+1}\right\|_{\hat{\Omega}_{j}}^{2}+\frac{c}{\varepsilon}\left\|\tilde{V}^{n+1}-V_{j}^{n+1}\right\|_{\hat{\Omega}_{j}}^{2} \\
& \leq(1+\varepsilon)\left\|U^{n+1}-\tilde{V}^{n+1}\right\|_{\hat{\Omega}_{j}}^{2}+\frac{c}{\varepsilon} e^{-\gamma L / \sqrt{\sigma}} \sigma\left\|\tilde{V}^{n+1}-V^{n}\right\|_{\Omega_{j}}^{2} .
\end{aligned}
$$

An analogous reasoning yields for the small subdomains

$$
\begin{aligned}
& \left\|U^{n+1}-V_{j, j+1}^{n+1}\right\|_{\hat{\Omega}_{j, j+1}}^{2}+\left\|U^{n+1}-V^{n+1}\right\|_{\Gamma_{j}}^{2} \\
& \leq(1+\varepsilon)\left(\left\|U^{n+1}-\tilde{V}^{n+1}\right\|_{\hat{\Omega}_{j, j+1}}^{2}+\left\|U^{n+1}-\tilde{V}^{n+1}\right\|_{\Gamma_{j}}^{2}\right) \\
& +\frac{c}{\varepsilon}\left(\left\|\tilde{V}^{n+1}-V_{j, j+1}^{n+1}\right\|_{\hat{\Omega}_{j, j+1}}^{2}+\left\|\tilde{V}^{n+1}-V^{n+1}\right\|_{\Gamma_{j}}^{2}\right) \\
& \leq(1+\varepsilon)\left(\left\|U^{n+1}-\tilde{V}^{n+1}\right\|_{\hat{\Omega}_{j, j+1}}^{2}+\left\|U^{n+1}-\tilde{V}^{n+1}\right\|_{\Gamma_{j}}^{2}\right) \\
& +\frac{c}{\varepsilon} e^{-\gamma L / \sqrt{\sigma}} \sigma\left(\left\|\tilde{V}^{n+1}-V^{n}\right\|_{\Omega_{j, j+1}}^{2}+\left\|\tilde{V}^{n+1}-V^{n}\right\|_{\Gamma_{j}}^{2}\right) .
\end{aligned}
$$

Now we plug $(27,28)$ into $(26)$ and get

$$
\begin{aligned}
E^{n+1} \leq & (1+\varepsilon)\left(\left\|U^{n+1}-\tilde{V}^{n+1}\right\|^{2}+\left\|U^{n+1}-\tilde{V}^{n+1}\right\|_{\Gamma}^{2}\right) \\
& +\frac{c}{\varepsilon} e^{-\gamma L / \sqrt{\sigma}} \sigma \sum_{j}\left(\left\|\tilde{V}^{n+1}-V^{n}\right\|_{\Omega_{j}}^{2}+\left\|\tilde{V}^{n+1}-V^{n}\right\|_{\Omega_{j, j+1}}^{2}+\left\|\tilde{V}^{n+1}-V^{n}\right\|_{\Gamma_{j}}^{2}\right) .
\end{aligned}
$$

The first term $U^{n+1}-\tilde{V}^{n+1}$ solves (10) with a functional $L^{n}(\Phi):=\left(U^{n}-V^{n}, \Phi\right)+\left(U^{n}-V^{n}, \Phi\right)_{\Gamma}$. Consequently, since the inequality of Poincaré-Friedrichs holds for both $\Omega$ and $\Gamma$,

$$
\left\|U^{n+1}-\tilde{V}^{n+1}\right\|^{2}+\left\|U^{n+1}-\tilde{V}^{n+1}\right\|_{\Gamma}^{2} \leq(1-c k)^{2} E^{n}
$$


is valid for sufficiently small time steps $k$, with a constant $c$ depending on $\kappa, \bar{\kappa}$. Further,

$$
\begin{aligned}
\sum_{j}\left(\left\|\tilde{V}^{n+1}-V^{n}\right\|_{\Omega_{j}}^{2}\right. & \left.+\left\|\tilde{V}^{n+1}-V^{n}\right\|_{\Omega_{j, j+1}}^{2}+\left\|\tilde{V}^{n+1}-V^{n}\right\|_{\Gamma_{j}}^{2}\right) \\
\leq & 2 \cdot\left(\left\|\tilde{V}^{n+1}-V^{n}\right\|^{2}+\left\|\tilde{V}^{n+1}-V^{n}\right\|_{\Gamma}^{2}\right) \\
\leq & c \cdot\left(\left\|\tilde{V}^{n+1}-U^{n+1}\right\|^{2}+\left\|\tilde{V}^{n+1}-U^{n+1}\right\|_{\Gamma}^{2}+\left\|U^{n+1}-U^{n}\right\|^{2}\right. \\
& \left.+\left\|U^{n+1}-U^{n}\right\|_{\Gamma}^{2}+\left\|U^{n}-V^{n}\right\|^{2}+\left\|U^{n}-V^{n}\right\|_{\Gamma}^{2}\right) .
\end{aligned}
$$

The last two terms right-hand side just form $E^{n}$, whereas the first two terms are bounded according to (30). The midst two terms are bounded by the data, using a standard spectral argument:

$$
\left\|U^{n+1}-U^{n}\right\|^{2}+\left\|U^{n+1}-U^{n}\right\|_{\Gamma}^{2} \leq c k^{2}
$$

Inserting all this into (29) we obtain

$$
E^{n+1} \leq\left((1+\varepsilon)(1-c k)^{2}+\frac{c}{\varepsilon} e^{-\gamma L / \sqrt{\sigma}} \sigma\right) E^{n}+\frac{c k^{2}}{\varepsilon} e^{-\gamma L / \sqrt{\sigma}} \sigma
$$

Now choose $\varepsilon \sim k$ and $L \sim \log \frac{1}{k}$ so that $e^{-\gamma L / \sqrt{\sigma}}=o\left(k^{3}\right)$ and

$$
E^{n+1} \leq E^{n}+c k^{4}
$$

results. This proves Theorem 2 .

\section{Proof of the Lemma 2}

We restrict ourselves to estimate (24), since (23) is part of [1]. As in the last proof we concentrate on the practically interesting case $(25)$. Let $d(x, y):=\operatorname{dist}\left((x, y), \partial \Omega_{j, j+1} \backslash \Sigma\right)$. Since $d \geq \delta$ in $\hat{\Omega}_{j, j+1}$ and on $\Gamma_{j}$, we have

$$
\begin{aligned}
\|U\|_{\hat{\Omega}_{j, j+1}}^{2}+\|U\|_{\Gamma_{j}}^{2} \leq & e^{-\gamma \delta / \sqrt{k}}\left\{\left(e^{\gamma d / \sqrt{k}} U, U\right)_{\Omega_{j, j+1}}+\left(e^{\gamma d / \sqrt{k}} U, U\right)_{\Gamma_{j}}\right. \\
& \left.+k \kappa\left(e^{\gamma d / \sqrt{k}} \nabla U, \nabla U\right)_{\Omega_{j, j+1}}+k \bar{\kappa}\left(e^{\gamma d / \sqrt{k}} U_{x}, U_{x}\right)_{\Gamma_{j}}\right\} .
\end{aligned}
$$

We neglect the index $\Omega_{j, j+1}$ in the following. Let $\Psi:=e^{\gamma d / \sqrt{k}} U$. Apparently, $\Psi_{I}-F \in X_{h}\left(\Omega_{j, j+1}\right)$ for its piecewise linear interpolate $\Psi_{I}$. Then

$$
\begin{aligned}
\left(e^{\gamma d / \sqrt{k}} U, U\right)+ & \left(e^{\gamma d / \sqrt{k}} U, U\right)_{\Gamma_{j}}+k\left(e^{\gamma d / \sqrt{k}} \kappa \nabla U, \nabla U\right)+k\left(e^{\gamma d / \sqrt{k}} \bar{\kappa} U_{x}, U_{x}\right)_{\Gamma_{j}} \\
= & \left(\Psi-\Psi_{I}, U\right)+\left(\Psi-\Psi_{I}, U\right)_{\Gamma}+k\left(\kappa \nabla\left(\Psi-\Psi_{I}\right), \nabla U\right) \\
& +k\left(\bar{\kappa}\left(\Psi-\Psi_{I}\right)_{x}, U_{x}\right)_{\Gamma_{j}}+(F, U)+(F, U)_{\Gamma_{j}}+k(\kappa \nabla F, \nabla U) \\
& +k\left(\bar{\kappa} F_{x}, U_{x}\right)_{\Gamma_{j}}-k\left(\kappa U \nabla\left(e^{\gamma d / \sqrt{k}}\right), \nabla U\right)-k\left(\bar{\kappa} U\left(e^{\gamma d / \sqrt{k}}\right)_{x}, U_{x}\right)_{\Gamma_{j}} .
\end{aligned}
$$

Each term right-hand side has to be estimated separately.

$$
\left(\Psi-\Psi_{I}, U\right) \leq \frac{c}{\varepsilon}\left(e^{-\gamma d / \sqrt{k}}\left(\Psi-\Psi_{I}\right), \Psi-\Psi_{I}\right)+\varepsilon\left(e^{\gamma d / \sqrt{k}} U, U\right) .
$$


Use elementwise-weighted $L^{2}$-estimates of the interpolation error to obtain

$$
\left(e^{-\gamma d / \sqrt{k}}\left(\Psi-\Psi_{I}\right), \Psi-\Psi_{I}\right) \leq c h^{4} e^{\gamma h / \sqrt{k}} \sum_{T \in \mathcal{T}} \int_{T} e^{-\gamma d / \sqrt{k}}\left|\nabla^{2} \Psi\right|^{2} \mathrm{~d} T .
$$

Since $e^{\gamma h / \sqrt{k}} \leq c$ and (elementwise $\Delta U=0$ )

$$
e^{-\gamma d / \sqrt{k}}\left|\nabla^{2} \Psi\right|^{2} \leq \frac{c \gamma^{2}}{k} e^{\gamma d / \sqrt{k}} \nabla U \cdot \nabla U+\frac{c \gamma^{2}}{k^{2}} e^{\gamma d / \sqrt{k}} U \cdot U
$$

we infer

$$
\left(\Psi-\Psi_{I}, U\right) \leq \frac{c \gamma^{2} h^{4}}{\varepsilon k^{2}}\left\{\left(e^{\gamma d / \sqrt{k}} U, U\right)+k\left(e^{\gamma d / \sqrt{k}} \kappa \nabla U, \nabla U\right)\right\}+\varepsilon\left(e^{\gamma d / \sqrt{k}} U, U\right)
$$

with some $\varepsilon$ to be fixed later. $\left(\Psi-\Psi_{I}, U\right)_{\Gamma_{j}}$ is estimated the same way, by observing that $\Psi_{I}$ is the piecewise linear interpolate of $\Psi$ on the interface. Consequently,

$$
\left(\Psi-\Psi_{I}, U\right)_{\Gamma_{j}} \leq \frac{c \gamma^{2} h^{4}}{\varepsilon k^{2}}\left\{\left(e^{\gamma d / \sqrt{k}} U, U\right)_{\Gamma_{j}}+k\left(e^{\gamma d / \sqrt{k}} \bar{\kappa} U_{x}, U_{x}\right)_{\Gamma_{j}}\right\}+\varepsilon\left(e^{\gamma d / \sqrt{k}} U, U\right)_{\Gamma_{j}}
$$

Further

$$
\begin{aligned}
& k\left(\kappa \nabla\left(\Psi-\Psi_{I}\right), \nabla U\right) \leq \frac{c \gamma^{2} h^{2}}{\varepsilon k}\left\{\left(e^{\gamma d / \sqrt{k}} U, U\right)+k\left(e^{\gamma d / \sqrt{k}} \kappa \nabla U, \nabla U\right)\right\}+\varepsilon\left(e^{\gamma d / \sqrt{k}} U, U\right) \\
& k\left(\bar{\kappa}\left(\Psi-\Psi_{I}\right)_{x}, U_{x}\right)_{\Gamma_{j}} \leq \frac{c \gamma^{2} h^{2}}{\varepsilon k}\left\{\left(e^{\gamma d / \sqrt{k}} U, U\right)_{\Gamma_{j}}+k\left(e^{\gamma d / \sqrt{k}} \bar{\kappa} U_{x}, U_{x}\right)_{\Gamma_{j}}\right\}+\varepsilon\left(e^{\gamma d / \sqrt{k}} U, U\right)_{\Gamma_{j}} .
\end{aligned}
$$

Consider now the terms containing $F$.

$$
(F, U)+(F, U)_{\Gamma_{j}} \leq \frac{c}{\varepsilon}\left(\|F\|^{2}+\|F\|_{\Gamma_{j}}^{2}\right)+\varepsilon\left(\left(e^{\gamma d / \sqrt{k}} U, U\right)+\left(e^{\gamma d / \sqrt{k}} U, U\right)_{\Gamma_{j}}\right)
$$

and

$$
\begin{aligned}
k(\kappa \nabla F, \nabla U)+k\left(\bar{\kappa} F_{x}, U_{x}\right)_{\Gamma_{j}} \leq & k \cdot\left\{\frac{c}{\varepsilon}\left(\kappa\|\nabla F\|^{2}+\bar{\kappa}\left\|F_{x}\right\|_{\Gamma_{j}}^{2}\right)\right. \\
& \left.\left.+\varepsilon\left(\left(e^{\gamma d / \sqrt{k}} \kappa \nabla U, \nabla U\right)+\left(e^{\gamma d / \sqrt{k}} \bar{\kappa} U_{x}, U_{x}\right)_{\Gamma_{j}}\right)\right)\right\}
\end{aligned}
$$

hold. And now the final terms, using $\left|\nabla e^{\gamma d / \sqrt{k}}\right| \leq \gamma / \sqrt{k} e^{\gamma d / \sqrt{k}}$

$$
\begin{aligned}
k\left(\kappa U \nabla\left(e^{\gamma d / \sqrt{k}}\right), \nabla U\right) & \leq \gamma \sqrt{k \kappa}\left(e^{\gamma d / \sqrt{k}} U, U\right)^{1 / 2}\left(e^{\gamma d / \sqrt{k}} \kappa \nabla U, \nabla U\right)^{1 / 2} \\
& \leq c \gamma\left(\left(e^{\gamma d / \sqrt{k}} U, U\right)+k\left(e^{\gamma d / \sqrt{k}} \kappa \nabla U, \nabla U\right)\right) \\
k\left(\bar{\kappa} U\left(e^{\gamma d / \sqrt{k}}\right)_{x}, U_{x}\right)_{\Gamma_{j}} & \leq \gamma \sqrt{k \bar{\kappa}}\left(e^{\gamma d / \sqrt{k}} U, U\right)_{\Gamma_{j}}^{1 / 2}\left(e^{\gamma d / \sqrt{k}} \bar{\kappa} U_{x}, U_{x}\right)_{\Gamma_{j}}^{1 / 2} \\
& \leq c \gamma\left(\left(e^{\gamma d / \sqrt{k}} U, U\right)_{\Gamma_{j}}+k\left(e^{\gamma d / \sqrt{k}} \bar{\kappa} U_{x}, U_{x}\right)_{\Gamma_{j}}\right) .
\end{aligned}
$$

Using (25) and choosing $\varepsilon$ and $\gamma$ sufficiently small, most terms are absorbed into the left-hand side of (33). Thus we plug (36-43) into (33) to obtain

$$
\begin{aligned}
\left(e^{\gamma d / \sqrt{k}} U, U\right)+ & \left(e^{\gamma d / \sqrt{k}} U, U\right)_{\Gamma_{j}}+k \kappa\left(e^{\gamma d / \sqrt{k}} \nabla U, \nabla U\right)+k \bar{\kappa}\left(e^{\gamma d / \sqrt{k}} U_{x}, U_{x}\right)_{\Gamma_{j}} \\
\leq & c\left\{\|F\|^{2}+\|F\|_{\Gamma_{j}}^{2}+k \kappa\|\nabla F\|^{2}+k \bar{\kappa}\left\|F_{x}\right\|_{\Gamma_{j}}^{2}\right\} .
\end{aligned}
$$


TABLE 1. Relative domain decomposition error, without or with one post-iteration step, as function of $i$ (one time step $k=i \cdot h^{2}, p=5, n=40, L=4$ ).

\begin{tabular}{||c|r|r|r|r|r||}
\hline$i$ & 1 & 2 & 3 & 4 & 5 \\
\hline$e($ iter $=0)$ & 4.08 & 13.10 & 27.10 & 45.00 & 49.80 \\
$e($ iter $=1)$ & 0.86 & 0.62 & 0.31 & 0.48 & 0.57 \\
\hline
\end{tabular}

Inserting this into (32) we conclude with

$$
\|U\|_{\hat{\Omega}_{j, j+1}}^{2}+\|U\|_{\Gamma_{j}}^{2} \leq c e^{-\gamma \delta / \sqrt{k}}\left\{\|F\|_{\Omega_{j, j+1}}^{2}+\|F\|_{\Gamma_{j}}^{2}+k \kappa\|\nabla F\|_{\Omega_{j, j+1}}^{2}+k \bar{\kappa}\left\|F_{x}\right\|_{\Gamma_{j}}^{2}\right\}
$$

Thus the estimate (24) and consequently the Lemma 2 is proved.

\section{Numerical TeStS}

In the present chapter we want to substantiate the theoretical results by numerical test computations. Let us approximate the function

$$
u(t, x, y)=(\sin t+\cos t)(\sin \pi x \cdot|\sin \pi p y|+1)
$$

in the total domain $\Omega:=(0,1)^{2}$, consisting of $p=J+1$ stripes $\Omega_{j}:=(0,1) \times\left(\frac{j-1}{p}, \frac{j}{p}\right) . u$ being continuous, its normal derivative jumps of size

$$
\left[u_{y}\right]=2 \pi p(\sin t+\cos t) \sin \pi x
$$

across all interfaces $\Gamma_{j}:=(0,1) \times\left\{\frac{\underline{p}}{\underline{p}}\right\} \cdot u$ solves the differential equations with the diffusion parameters $\kappa=\bar{\kappa}=$ 0.01 and suitable source terms $f, \bar{f}$ and initial data. We do not care about the nonzero boundary data. Each $\Omega_{j}$ is a half-periodicity cell, discretized by $n \times n$ rectangular elements, $h:=\frac{1}{n}$ denoting the mesh size. All the linear algebraic systems are solved by the diagonally preconditioned $\mathrm{cg}$-method.

In the computations we use the following values, if not stated else: $p=5, n=40$, time step $k=h^{2}$ (to have a second order scheme), and $L=4$ rows of overlapping elements. In order to make the results comparable we present the outcomes of one time step in terms of the relative domain decomposition error, i.e. the ratio

$$
e:=\frac{\|(V-U)(t, .)\|}{\|(U-u)(t, .)\|}
$$

It suffices to show this $L^{2}$-error, since the corresponding $L^{\infty}$-error (reflecting the averaging process in the overlapping area) generally behaves similarly, as several experiments have shown. Of practical interest are equilibrated values, i.e. parameters where $e \sim 1$.

In Figure 5 we investigate the error as a function of both $n$ and $L$. We see that, for a given relative error $e$, the overlap $L$ increases 'nearly logarithmically' with $n$, as expected from Theorem 2 ( $c f$. the numerical results by $[1,10])$. - Moreover, Figure 6 shows that the error due to domain splitting, considered as a function of time, increases essentially weaker than the pure discretization error. This interesting feature (observed previously in [10]) implies a chance to further decrease the overlap size $L$ in time. This would help to reduce the parallel overhead. - Nevertheless, in some cases, the overlap may be inconvenient or too large. It may be quite often drastically reduced by iterating locally on our algorithm. This shows up in Table 1 where we consider the error as a function of $i:=k / h^{2}$. In fact, such iterations serve to improve on the boundary data on the artificial inner boundaries. Note that, to this end, the local problems have been solved only once, consequently the additional expenses are low. A different approach, a higher order extrapolation in time of such boundary data, has been employed by [1] (for the Crank-Nicholson time discretization scheme). 


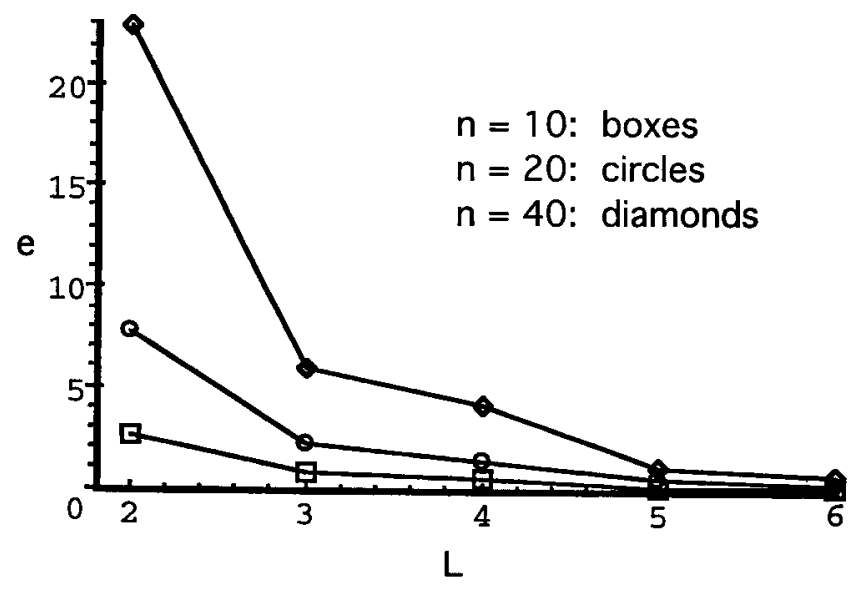

Figure 5. Relative domain decomposition error as function of $L$ and $n . n=10$ : boxes, $n=20$ : circles, $n=40$ : diamonds (one time step $k=h^{2}, p=5$ ).

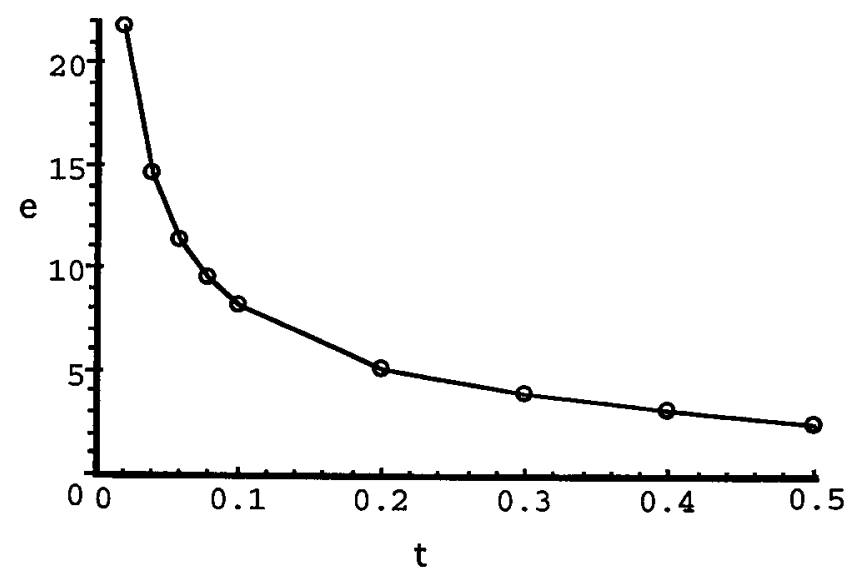

Figure 6. Relative domain decomposition error as function of time $\left(p=5, n=40, k=4 h^{2}\right.$, $L=4)$.

In the final experiment we ask how the error depends on the number $p$ of subdomains. Due to storage requirements we choose the parameters $n=20, L=3$. In Figure 7 it shows up that the relative domain decomposition error and so the required overlap stays 'nearly constant' with $p$. These parameters are thus appropriate for investigating the scalability of our algorithm by inspecting the CPU times. In all time measurements we neglect the solver, since our preconditioner of the $c g$-method is certainly suboptimal (which is not among the topics of the present paper, $c f$. also [9]). We thus measure the CPU times, without or with domain splitting, for establishing the stiffness matrices and the right-hand sides of the algebraic systems (and also for averaging the patchwise solutions to a single-valued function). Let $T_{1}(p)$ denote the CPU time (in seconds) when handling $p$ subdomains on one processor with the global algorithm, and $T_{p}(p)$ and $E(p)=T_{1}(p) /\left(p T_{p}(p)\right)$ the average CPU time or the parallel efficiency respectively when handling $p$ subdomains on $p$ processors with the domain splitting scheme. We simulate in fact all processors on a single one, thus neglecting the processor communication times. Table 2 shows clearly that the proposed domain splitting scheme nearly scales with respect to the number of subdomains. - It should be noticed that, whereas the arithmetic overhead due to our overlapping domain splitting is generally low on fine meshes (the case of practical interest), it is not negligible for 


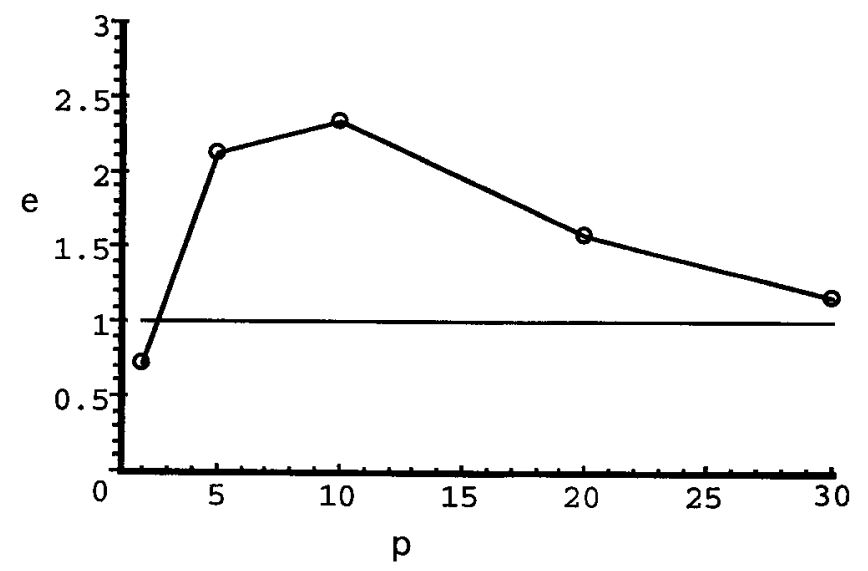

FiguRE 7. Relative domain decomposition error as function of $p$ : circles (one time step $k=h^{2}$, $n=20, L=3)$.

TABLE 2. Parallel efficiency $E(p)$ of the domain splitting method (without communication times), as function of $p$ (one time step $k=h^{2}, n=20, L=3$ ).

\begin{tabular}{||c|r|r|r|r|r||}
\hline $\begin{array}{c}p \\
\text { \# unknowns }\end{array}$ & 2 & 4 & 8 & 16 & 32 \\
\hline$T_{1}(p)$ & 0.427 & 0.893 & 1.878 & 3.783 & 7.453 \\
$T_{p}(p)$ & 0.235 & 0.257 & 0.277 & 0.282 & 0.280 \\
\hline$E(p)$ & 0.91 & 0.87 & 0.85 & 0.84 & 0.83 \\
\hline
\end{tabular}

the present data: if measured in terms of the additional degrees of freedom, we expect $E_{p} \approx n /(n+(2 L-1))=0.8$ for large $p$. This fits well with our experimental results. Note also that, since the domain splitting algorithm is essentially noniterative, the processor communication times are expected low. This important point should further be addressed with a physical example and employing a parallel computer.

The author expresses sincere thanks to Professors K.-H. Hoffmann (Bonn) and Yu.A. Kuznetsov (Houston) for many discussions on composite materials and domain decomposition methods. Financial support by the University of Houston and the DFG-Sonderforschungsbereich 'Mathematische Modellierung, Simulation und Verifikation in materialorientierten Prozessen und intelligenten Systemen' at the Technische Universität München is gratefully acknowledged.

\section{REFERENCES}

[1] H. Blum, S. Lisky and R. Rannacher, A domain splitting algorithm for parabolic problems. Computing 49 (1992) 11-23.

[2] D. Braess, W. Dahmen and Chr. Wieners, A multigrid algorithm for the mortar finite element method. SIAM J. Numer. Anal. 37 (1999) 48-69.

[3] H. Chen and R.D. Lazarov, Domain splitting algorithm for mixed finite element approximations to parabolic problems. EastWest J. Numer. Math. 4 (1996) 121-135.

[4] Z. Chen and J. Zou, Finite element methods and their convergence analysis for elliptic and parabolic interface problems. Numer. Math. 79 (1998) 175-202.

[5] W. Hackbusch, Theorie und Numerik elliptischer Differentialgleichungen. Teubner, Stuttgart (1986).

[6] W. Hackbusch and S. Sauter, Composite finite elements for the approximation of PDEs on domains with complicated microstructures. Numer. Math. 75 (1997) 447-472.

[7] H. Haller, Composite materials of shape-memory alloys: micromechanical modelling and homogenization (in German). Ph.D. thesis, Technische Universität München (1997). 
[8] F.H. Hebeker, An a posteriori error estimator for elliptic boundary and interface problems. Preprint 97-46 (SFB 359), Universität Heidelberg (1997); submitted.

[9] F.K. Hebeker, Multigrid convergence analysis for elliptic problems arising in composite materials (in preparation).

[10] F.K. Hebeker and Yu.A. Kuznetsov, Unsteady convection and convection-diffusion problems via direct overlapping domain decomposition methods. Preprint 93-54 (SFB 359), Universität Heidelberg, 1993; Numer. Methods Partial Differential Equations 14 (1998) 387-406.

[11] K.H. Hoffmann and J. Zou, Finite element analysis on the Lawrence-Doniach model for layered superconductors. Numer. Funct. Anal. Optim. 18 (1997) 567-589.

[12] J. Jäger, An overlapping domain decomposition method to parallelize the solution of parabolic differential equations (in German). Ph.D. thesis, Universität Heidelberg (1994).

[13] C. Kober, Composite materials of shape-memory alloys: modelling as layers and numerical simulation (in German). Ph.D. thesis, Technische Universität München (1997).

[14] Yu.A. Kuznetsov, New algorithms for approximate realization of implicit difference schemes. Sov. J. Numer. Anal. Modell. 3 (1988) 99-114.

[15] Yu.A. Kuznetsov, Domain decomposition methods for unsteady convection diffusion problems. Comput. Methods Appl. Sci. Engin. (Proceedings of the Ninth International Conference, Paris 1990) SIAM, Philadelphia (1990) $211-227$.

[16] Yu.A. Kuznetsov, Overlapping domain decomposition methods for finite element problems with singular perturbed operators. in Domain decomposition Methods for Partial differential equations, R. Glowinski et al. Eds., SIAM, Philadelphia. Proc. of the 4th Intl. Symp. (1991) 223-241

[17] A. Quarteroni and A. Valli, Numerical Approximation of Partial Differential Equations. Springer, Berlin etc. (1994).

[18] R. Rannacher and J. Zhou, Analysis of a domain splitting method for nonstationary convection-diffusion problems. East-West J. Numer. Math. 2 (1994) 151-172.

[19] J. Wloka, Partielle Differentialgleichungen. Teubner, Stuttgart (1982). 